\title{
Detailed Observations of a Burst of Energetic Particles in the Deep Magnetotail by Geotail
}

\author{
E. T. SARRIS ${ }^{1,2}$, V. ANGELOPOULOS ${ }^{3}$, R. W. MCENTIRE ${ }^{3}$, D. J. WILliams ${ }^{3}$,

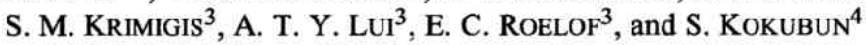 \\ ${ }^{1}$ Institute of Space Research, National Observatory of Athens, Greece \\ ${ }^{2}$ Demokritos University of Thrace, Xanthi, Greece \\ ${ }^{3}$ Applied Physics Laboratory, The Johns Hopkins University, Laurel, MD, U.S.A. \\ ${ }^{4}$ STELAB, Nagoya University, Toyokawa 442, Japan
}

(Received August 17, 1995; Accepted May 28, 1996)

\begin{abstract}
Using data from the energetic particle detector on board the GEOTAIL satellite we report on a case study of electron and ion bursts observed at $X_{\mathrm{GSM}}=-128 \mathrm{R}_{\mathrm{E}}$. Based on the arrival times of energetic electrons, protons, Helium and Oxygen, as well as the time of flight channels designed specifically for remote sensing purposes, we estimate that the particle acceleration took place at $X_{\mathrm{GSM}} \cong-103 \mathrm{R}_{\mathrm{E}}$. The ensuing tailward particle anisotropy gives a bulk flow estimate that is consistent with the arrival time of the plasma from the expected location of the acceleration region.
\end{abstract}

\section{Introduction}

One of the most important subjects of Solar-Terrestrial Plasma Physics is the study of the acceleration processes, by which a fraction of the plasma particles receives a large part of the available free energy, resulting in the formation of superthermal tails in the particle population. In the earth's magnetosphere these processes are manifested by the occurrence of bursts of energetic particles $(E>30 \mathrm{keV})$ observed by a series of spacecraft in the near earth magnetotail up to distances of $\sim 45 R_{E}$ (e.g., Krimigis and Sarris (1979), and references therein).

The identification of the source and the acceleration mechanism, which gives rise to the non-thermal particle population in the magnetotail, have been explored incorporating fine-time resolution observations by a number of spacecraft. In particular, the analysis of velocity dispersion effects during the onsets of bursts of energetic particles has been utilized to infer the location of the acceleration source and to provide estimates of the growth rate of the induced transient electric fields (Sarris et al., 1976; Kirsch et al., 1977, 1981; Williams, 1981; Sarafopoulos et al., 1984; Sergeev et al., 1986; Sarafopoulos and Sarris, 1987, 1988; Pavlos et al., 1989).

A distinct characteristic of the onsets of high intensity impulsive ion $(E>50 \mathrm{keV})$ bursts, which arc observed in the well explored region of the magnetotail (up to $\sim 40 \mathrm{R}_{\mathrm{E}}$ ), has been reported to be the display of Inverse Velocity Dispersion (IVD), whereby low energy particles are detected prior to the high energy ones (Sarris et al., 1976). The IVD effect observed during ion bursts at the plasma sheet boundary was explained as the result of rapid inward convection of boundary field lines (Sarris and Axford, 1979). However the frequent occurrence of IVD during particle bursts detected deep inside the plasma sheet, as well as at the plasma sheet boundary during periods of both inward and outward convection, has led to the suggestion that the IVD effect is the display of the temporal evolution of the accelerating electric field, i.e. the growth rate of the responsible plasma instability (Sarafopoulos and Sarris, 1988). A number of model calculations of the acceleration of particles by the inductive electric field produced by an explosivc magnetic perturbation have provided results in agreement with the above IVD observations (Taktakishvili and Zelenyi, 1988, 1990; Zelenyi et al., 1990). 
Along these lines it is anticipated that the fingerprint of the IVD effect should be preserved in the vicinity of the acceleration source. However, beyond a critical distance the high energy particles would overtake the low energy ones and no IVD should be observed. On the basis of a survey of observations of impulsive bursts of energetic ions by the IMP spacecraft (at circular orbits $\sim 35$ to $\sim 45 R_{E}$ ), which showed that the IVD effect was a common feature of these events, Sarafopoulos and Sarris (1988) concluded that the acceleration source is frequently located in the magnetotail within a distance of $\sim 80 \mathrm{R}_{\mathrm{E}}$ from the Earth. Furthermore, the presence of a semi-permanent source of energetic particles in the distant magnetotail at $\sim 100 \mathrm{R}_{\mathrm{E}}$ was inferred on the basis of near-earth observations by Williams (1981).

In this work we analyze in detail the impulsive onset of an energetic particle burst event detected by the EPIC instrument on board the GEOTAIL spacecraft at a distance of $X_{\mathrm{GSM}}=128 \mathrm{R}_{\mathrm{E}}$ in the magnetotail. From the time delays between the onset of the energetic electron pulse and the onsets of the various species of the energetic ions as well as from the inferred plasma flow velocity we find that a self-consistent picture arises if the acceleration region was $\sim 100 R_{E}$ away from Earth.

\section{Instrumentation and Data Sets}

Measurements of energetic ions and electrons were obtained by the ion composition sensor (ICS) of the energetic particle and ion composition (EPIC) instrument on board the GEOTAIL satellite (Williams et al., 1994). The satellite is spinning approximately on the solar ecliptic plane. The ICS sensor provides ion composition, energy spectra and directional intensities in 16 azimuthal sectors of $22.5^{\circ}$, over two polar angles (North Head: $8^{\circ}$ to $38^{\circ}$, South Head: $-8^{\circ}$ to $-38^{\circ}$ ) for energies from $50 \mathrm{keV}$ to $3 \mathrm{MeV}$. Each of the ion-heads employs an electron sweeping magnet and provides measurements of both the time-of-flight (TOF) and the energy accumulation solid state detector (SSD). In addition, ICS provides electron measurements in the energy range of $34-110 \mathrm{keV}$ in one plane $\left(-30^{\circ}\right.$ to $30^{\circ}$ elevation) and in 8 equal azimuthal sectors.

For the purposes of this paper we utilized the following ICS channels: E3, E4, E5, He2, M2, T1 1 and T14. Table 1 presents an overview of the channel characteristics. E3, E4 and E5 are SSD channels in the 62-74 keV, 74-89 keV and 89-110 keV energy range respectively, without mass discrimination but with much higher count rates than the individual species channels. He2 represents Helium species and M2 represents mostly Oxygen. The time-of-flight (TOF) channels provide the differential flux of ions of a given velocity within the detector. Making an assumption regarding the dominant species we can find the energy of the incident ions (external velocity) for each TOF channel. Two of the TOF channels return high time resolution data: T11 and T14 at $6 \mathrm{sec}$ and $3 \mathrm{sec}$ resolution respectively. Assuming that the ions detected are protons (an assumption that is substantiated by the pulse height analysis data for the period of interest), T11 and T14 correspond to incident proton energies of $35-45 \mathrm{keV}$ and $15-20 \mathrm{keV}$ respectively. Finally, we use the electron measurements from channel ED1 that corresponds to energies of $>34 \mathrm{keV}$.

Table 1. EPIC/ICS channels.

\begin{tabular}{lclc}
\hline Channel & Resolution & \multicolumn{1}{c}{ Description } & Incident velocity $(\mathrm{km} / \mathrm{s})$ \\
\hline ED 1 & $3 \mathrm{~s}$ & Electrons $>34 \mathrm{keV}$ & Assume $\pm \infty$ \\
E 3 & $6 \mathrm{~s}$ & All ions of $62-74 \mathrm{keV}\left(\right.$ Mostly $\left.\mathrm{H}^{+}\right)$ & $3600 \pm 150$ \\
E 4 & $6 \mathrm{~s}$ & All ions of $74-89 \mathrm{keV}($ Mostly H) & $3950 \pm 200$ \\
E 5 & $3 \mathrm{~s}$ & All ions of $89-110 \mathrm{keV}($ Mostly H & $4350 \pm 250$ \\
He2 & $6 \mathrm{~s}$ & Helium of $70-96 \mathrm{keV}($ Mostly He & $2000 \pm 150$ \\
M2 & $6 \mathrm{~s}$ & Oxygen of $187-222 \mathrm{keV}\left(\right.$ Mostly $\left.\mathrm{O}^{+}\right)$ & $1600 \pm 100$ \\
T11 & $3 \mathrm{~s}$ & All ions with TOF $28-34$ ns (Mostly $\left.\mathrm{H}^{+}\right)$ & $2700 \pm 100$ \\
T14 & $6 \mathrm{~s}$ & All ions with TOF 50-60 ns (Mostly $\left.\mathrm{H}^{+}\right)$ & $1830 \pm 150$ \\
\hline
\end{tabular}


In addition, we used information from the supra-thermal ion composition spectrometer (STICS) detector of the EPIC instrument. STICS provides $\sim 4 \pi$ angular coverage, composition and charge state measurements for ions of 30-230 keV/e, and mass per charge measurements for ions $>7.5 \mathrm{keV} / \mathrm{e}$ (Williams et al., 1994). We utilized STICS' ability to discriminate ion charge states as well as its 3-dimensionality. According to measurements from STICS, the dominant species for the event under study was $\mathrm{H}^{+}$; thus we assumed that E3, E4 and E5 are effectively protons. In addition, the predominant charge state of Helium was $\mathrm{He}^{++}$and that of $\mathrm{Oxygen}$ was $\mathrm{O}^{+}$.

The magnetic field was measured by the magnetic field experiment (MGF) on board GEOTAIL with a $\pm 0.1 \mathrm{nT}$ sensitivity in the $X$ and $Y$ spacecraft coordinate axes and with a $\pm 0.3 \mathrm{nT}$ sensitivity in the $Z$ axis (Kokubun et al., 1994).

\section{Observations and Analysis}

An isolated, highly anisotropic burst of tailward streaming particles was detected at $~ 1800 \mathrm{UT}$ on March 30, 1993, following an extended period of low particle intensities and undisturbed magnetic field conditions. Figure 1 displays an overview of the anisotropy of the energetic electrons (channel ED 1: $E>$ $34 \mathrm{keV}$ ), of the omnidirectional ion intensity spectrum and of the energetic ion anisotropy (channel E3, primarily protons: $62-74 \mathrm{keV}$ ) for a two-hour period (17:30-19:30 UT) encompassing the event. The anisotropy plots show the particle differential flux in the azimuthal direction $(\phi)$ in the GSE system. Measurements ( $3 \mathrm{~s}$ averages) of the magnetic field components and magnitude appear in the top four panels in the GSM system. The burst of energetic particles started with an impulsive onset and lasted for $\sim 40$ min following a slow decay to the level of the ambient particle intensities. In the magnetic field vector display it is seen that the prevailing quiet field conditions were disrupted by an impulsive MHD perturbation, prominent in both the field magnitude and direction. The disturbed magnetic field conditions continued well after the decay of the particle burst event.

The abrupt onset of the electron and ion intensities is shown in greater detail in an expanded display covering the period 17:57-18:17 UT. Figure 2 shows both color anisotropy plots for 16 sectors and line plots of the 4-sector averaged directional intensities of energetic electrons (lower two panels) and ions (next two panels up), along with the magnetic field magnitude and $B_{X}, B_{Y}, B_{Z}$ components (top three panels). The azimuthal angle of the field is also displayed in one of the color anisotropy plots (E3 channel). Highly collimated field-aligned tailward streaming was observed at the onset of the event for both the energetic electrons and ions, whereas a moderately anisotropic antisunward flux increase started at 18:08:30 UT at a large angle to the magnetic field, since the $B_{X}$ component was the least significant during that period.

Distinct velocity dispersion effects are clearly seen at the onset of the event. The impulsive onset of the streaming energetic electrons was observed at 18:00:05 UT and that of the ions at 18:00:50 UT. As noted above the magnetic field remained quiet until 18:07:30 UT, which marked the abrupt onset of the prominent magnetic field disturbance. Furthermore the sharp change in the ion flux anisotropy observed at 18:08:30 UT, which clearly marked the onset of less anisotropic ion intensities (typical forward to backward ratios of 2 to 5), is indicative of a convective-type flow of the bulk plasma population.

Measurements of the ion intensities in channel E4 (74-89 keV) with the same time resolution (3 s) show an onset time $\sim 6 \mathrm{~s}$ earlier than the event onset in channel E3. Additional energy and species channels, which are available for timing of the burst onset with good resolution are presented in Fig. 3. Table 2 summarizes our calculations using the timing information from Fig. 3. The lower time resolution (48-96 s) and/or counting rates at the higher energy ICS channels do not permit an accurate determination of the event onset for the detection of possible dispersive effects in connection with the lower energy channels. However, indications for the presence of velocity dispersion effects are also present in measurements of the ICS species channels $\mathrm{He} 2(70-96 \mathrm{keV}$ ) and M2 (Oxygen: 187-222 keV), despite the low counting rates in these channels. The onset of the $\mathrm{He} 2$ intensities is detected within 18:01:00-18:01:40 UT and that of the Oxygen intensities within 18:01:40-18:02:20 UT. 
GEOTAIL, 1993-Mar-30, GSM Position: (-128.2, 9.8, 3.8)
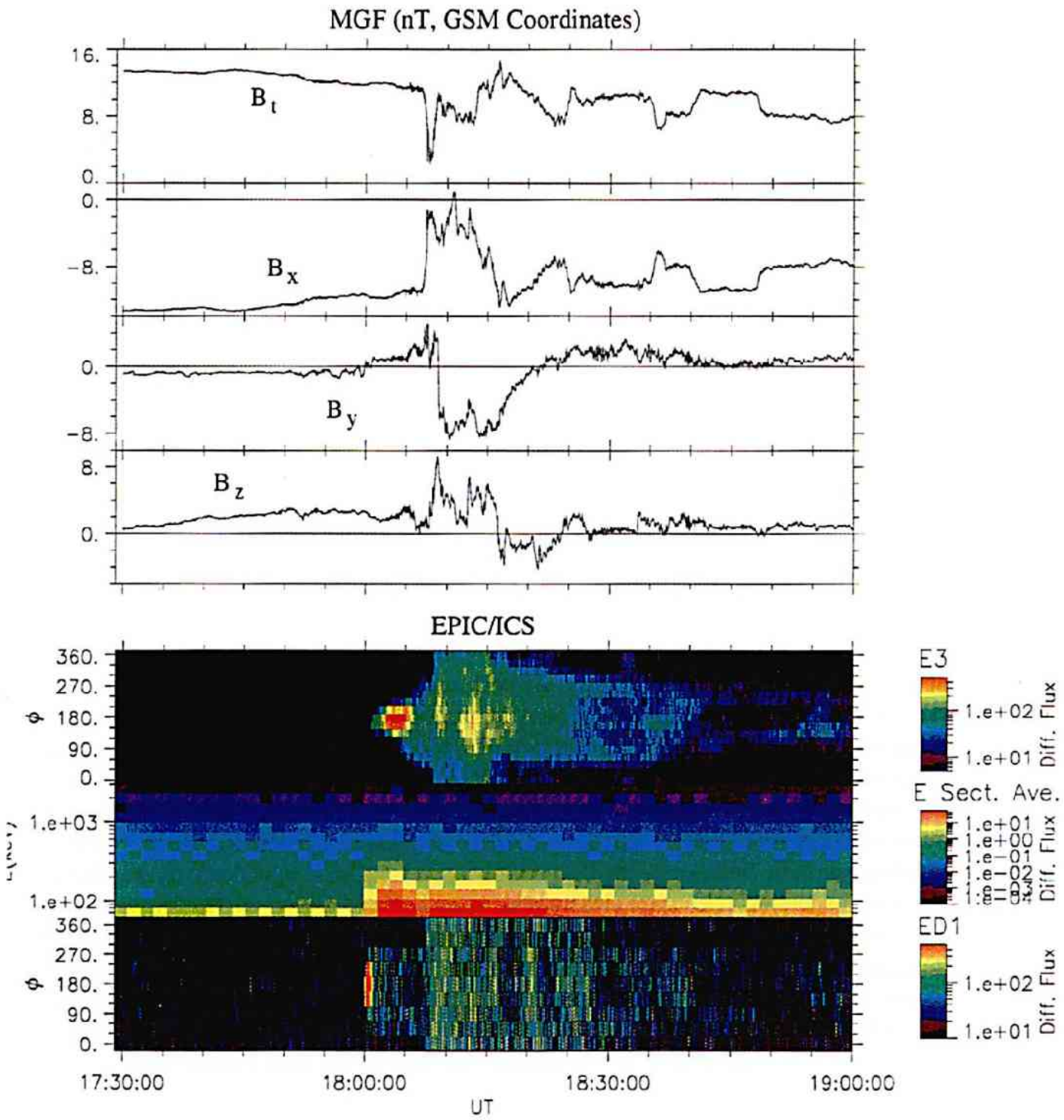

Fig. 1. A 1.5 hour overview of the magnetic field and energetic particle conditions around the energetic particle burst under study. The burst occurred at 1800 UT. The top 4 panels show the magnetic field in GSM coordinates at 3 s resolution. The bottom 3 color panels show the differential energy fluxes of: 1) the energetic ion channel E3 in a $\mathrm{f}-\mathrm{UT}$ format, where $\mathrm{f}$ is the GSE longitude of the particle direction. 2) the sector averaged ion channels in an E-UT format (spectrogram), where E is the particle energy. 3) the energetic electron channel EDl in a f-UT format. The resolution is $6 \mathrm{~s}$ for E3, $96 \mathrm{~s}$ for the ion spectrogram and $3 \mathrm{~s}$ for ED1. We interpret ions in the energy channels as protons based on results from the STICS/EPIC head. 

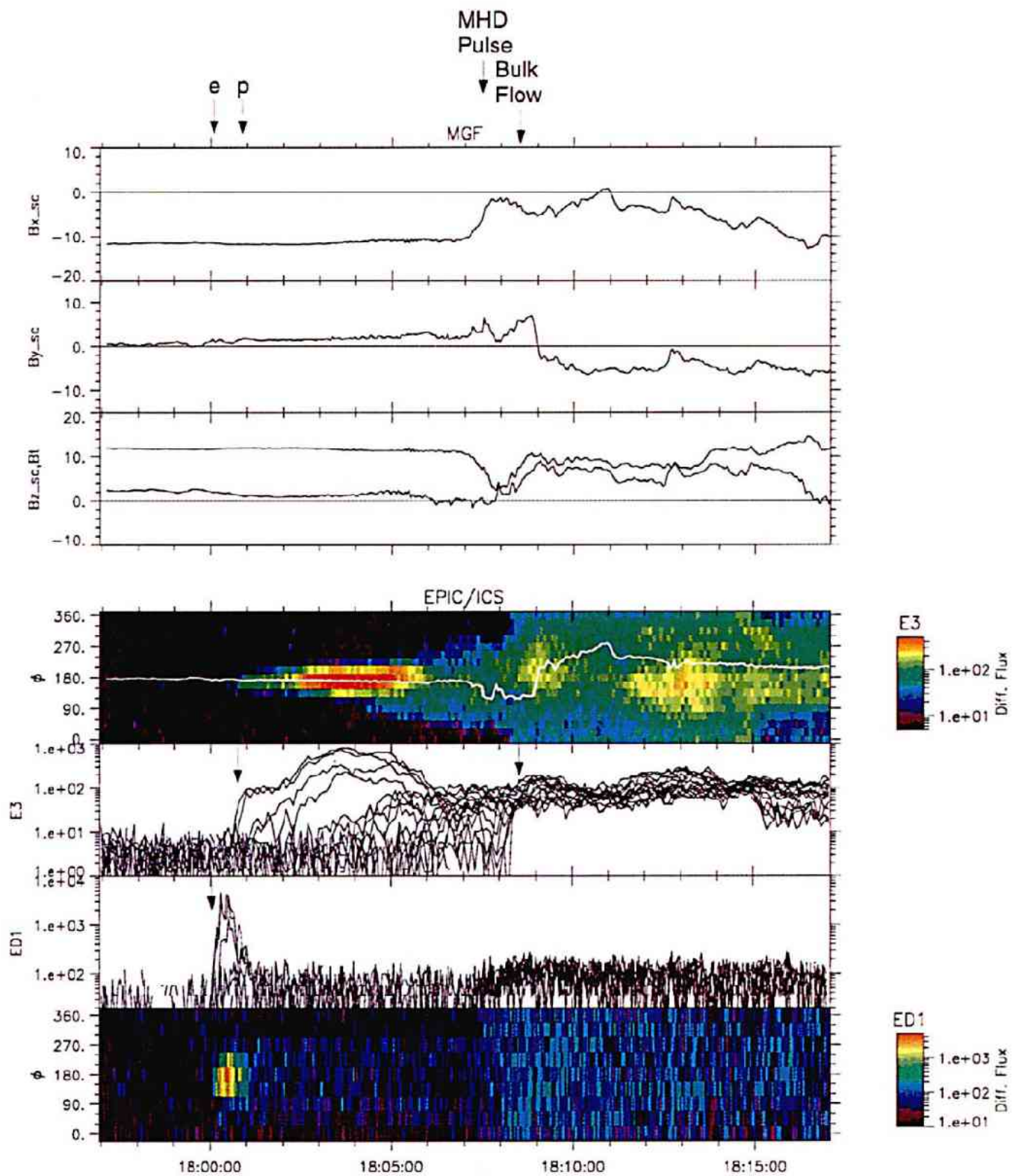

Fig. 2. An expanded view of the time of the energetic ion and electron bursts (17:57-18:17 UT). The color panels are simply detailed versions of the f-UT panels of Fig. 1 for ions and electrons respectively. The line plots are directional intensities of all sectors of the ions (panel 6) and the electrons (pancl 7). Intense ficld-aligned electron and ion streaming with distinct velocity dispersion features is detected at the onset of the event, which is followed by an abrupt magnetic disturbance and a convective-type bulk flow. 


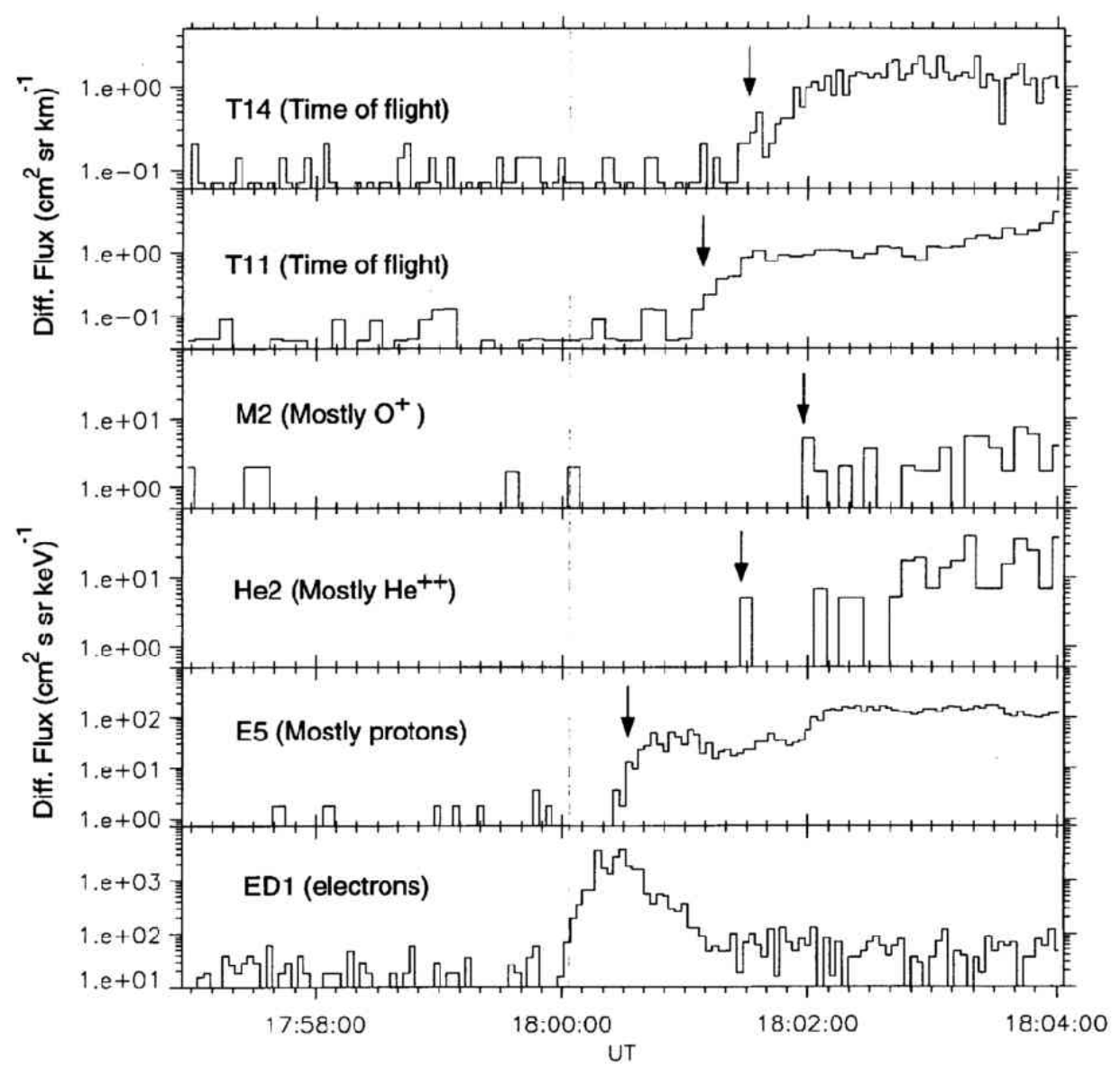

Fig. 3. Differential energy flux measured in the antisunward sector of several channels (see Table 1) of the EPIC/ICS head. The dispersion seen in different channels is used in Table 2 to get an estimate of the distance to the energization location assuming all particles came from the same place.

Table 2. Source-distance calculations for the sequence of onsets.

\begin{tabular}{lccc}
\hline Channel & $D t$ (seconds) & Incident velocity $V(\mathrm{~km} / \mathrm{s})$ & $D s=D t \cdot V\left(\mathrm{R}_{\mathrm{E}}\right)$ \\
\hline E3 & $45 \pm 6$ & $3600 \pm 100$ & $25 \pm 3$ \\
E5 & $29 \pm 6$ & $4350 \pm 250$ & $20 \pm 5$ \\
He2 & $82 \pm 9$ & $2000 \pm 150$ & $26 \pm 5$ \\
T11 & $112 \pm 9$ & $1600 \pm 100$ & $28 \pm 4$ \\
T14 & $67 \pm 9$ & $2700 \pm 100$ & $28 \pm 5$ \\
& $90 \pm 6$ & $1800 \pm 150$ & \\
\hline MHD pulse & $445 \pm 10 \mathrm{~s}$ & inferred speed: $360 \pm 10$ & \\
\hline Bulk flow & $505 \pm 10 \mathrm{~s}$ & inferred speed: $310 \pm 10$ & \\
\hline \multicolumn{4}{l}{ Convective bulk flow from compton-getting: $300 \pm 30$} \\
\hline
\end{tabular}




\section{Discussion}

The sequence of events described above during the dispersive onset of the burst of energetic particles, which was detected by Geotail in the deep magnetotail at a distance $\sim 129 \mathrm{R}_{\mathrm{E}}$ from the Earth on March 30 , 1993, is summarized in Table 2.

The observed distinct velocity dispersion effects contain important information on the location of the source of energetic particles and the dynamics of the magnetotail and will be analyzed in detail under certain assumptions. A possible interpretation of the dispersive onsets of particle bursts in the magnetotail has been given in terms of the Boundary Layer Model (Richardson et al. (1993), and references therein). In the following we proceed to test self-consistently the alternative hypothesis that the dispersive onset of the particle burst, as well as the sequence of the MHD pulse and the onset of the bulk flow of superthermal particles are temporal signatures and not the result of the crossing of a spatial structure or boundary. Under the above hypothesis it is suggested that the energetic electrons and ions were accelerated by the inductive electric fields developed during the explosive phase of a plasma instability, which initiated the impulsive MHD disturbance and gave rise to the bulk flow of the superthermal particles. Each of the above presumably propagated from the source site to the location of the spacecraft with a different speed.

The fast energetic electrons $\left(V_{\mathrm{e}}=82,000 \mathrm{~km} / \mathrm{s}\right)$ streaming tailward along the field reached GEOTAIL first followed by the considerably slower energetic ions of channel E3 $\left(V_{\mathrm{p}}=3570 \mathrm{~km} / \mathrm{s}\right)$. From the measured time delay $T_{\mathrm{ei}}=45 \mathrm{~s}$ between the electron and ion event onsets we estimate that the source-site was located at a distance $\sim 160,000 \mathrm{~km}$ (i.e. $\sim 25 \mathrm{R}_{\mathrm{E}}$ ) earthward of the GEOTAIL spacecraft. The travel time of the energetic electrons $(\sim 2 \mathrm{sec})$ is below the instrument resolution, therefore the onset of the impulsive electron burst at the spacecraft can be considered as nearly identical with the onset of the events at the source.

The sharp MHD pulse detected at GEOTAIL is thought to originate in the explosive magnetic disturbance giving rise to the large inductive electric fields, which accelerated the energetic electrons and ions. Using the above estimate of the source distance and the measured time delay $T_{\mathrm{eM}}=445 \mathrm{~s}$ between the onset time of the electron burst and that of the impulsive disturbance in the magnetic field we compute the speed of the MHD pulse $V_{\mathrm{MHD}}=360( \pm 10) \mathrm{km} / \mathrm{s}$, which was presumably launched from the sourcesite at the same time with the energetic particles and propagated to the spacecraft along the magnetic field. Since for propagation along the field both the fast and the intermediate modes travel at the Alfvén speed, we deduce that in this region of the distant magnetotail $V_{\mathrm{A}} \sim V_{\mathrm{MHD}}=360 \mathrm{~km} / \mathrm{s}$.

If the sharp onset of the less anisotropic ion fluxes at 18:08:30 Ut is indeed the mark of the arrival of the bulk flow of the superthermal plasma population, which was also originated at the source site at the time of the explosive phase of the plasma instability, then using the inferred distance to the source and the measured time delay, the bulk flow speed is estimated to be $\sim 310( \pm 20) \mathrm{km} / \mathrm{s}$. The convective (bulk) speed of the superthermal particle population after 18:08:30 UT can also be estimated indirectly from the Compton-Getting effect (Gold et al., 1975). From measurements of the spectral index $(\gamma \sim 2.3)$ and the anisotropy ratio $\left(j_{\mathrm{f}} / j_{\mathrm{b}}=3\right)$ at the time of the onset, the Compton-Getting transformation gives a bulk flow speed $V_{\text {bulk }} \sim 300 \mathrm{~km} / \mathrm{s}$, which is in good agreement with the above estimate.

All the relevant physical parameters, which were derived under the above assumptions, are included in Table 2. It is remarkable that all channels give an approximate but quite consistent source location of $20-28 \mathrm{R}_{\mathrm{E}}$. Even for the $\mathrm{He}^{++}$and $\mathrm{O}^{+}$species channels for which counting statistics are not very good due to the small fluxes observed at the time, the first appearance of above-background ion fluxes (18:01:0018:01:40 UT for channel He2 and 18:01:40-18:02:20 UT for channel M2) gives a source location in agreement with the calculations done using channels with better statistics.

It is concluded that the sequence of events during the dispersive onset of the particle burst observed by Geotail at $\sim 128 \mathrm{R}_{\mathrm{E}}$ presents a self-consistent picture in the framework of the above analysis, which in this case indicates the presence of an impulsive source of energetic particles in the magnetotail at a distance 
$\sim 103 \mathrm{R}_{\mathrm{E}}$ from the earth. The presence of a semi-permanent source of energetic particles in the distant magnetotail at $\sim 100 R_{E}$ was inferred indirectly on the basis of near-earth observations by Williams (1981). Thus the distant magnetotail appears to be also an active part in the explosive particle acceleration processes and not just a passive channel of such activity from the near earth magnetosphere.

We wish to thank S. P. Christon and D. H. Fairfield for their assistance in the processing of the particle and magnetic field data respectively. We are grateful to S. Nylund, M. Grimmes, and P. Foltz for their programming help during the course of this paper. APL support for the EPIC/GEOTAIL effort resulted from NASA Space Physics Division under Dept. of Navy Task IAFX9BXX; Contract N00039-91-C-001.

\section{REFERENCES}

Gold, R. E., C. O. Bostrom, and E. C. Roelof, Anisotropy measurements of $\sim 50 \mathrm{keV}$ solar protons, Conf. Pap. Int. Cosmic Ray Conf. 14th, 5, 1801, 1975.

Kirsch, E., S. M. Krimigis, E. T. Sarris, R. P. Lepping, and T. P. Armstrong, Possible evidence for large transient electric fields in the magnetotail from oppositely directed anisotopies of energetic protons and electrons, Geophys. Res. Lett., 4, 137-140, 1977.

Kirsch, E., S. M. Krimigis, E. T. Sarris, and R. P. Lepping, Detailed study on acceleration and propagation of energetic protons and electrons in the magnetotail during substorm activity, J. Geophys. Res., 86, 6727-6738, 1981.

Kokubun, S., T. Yamamoto, M. H. Acuna, K. Hayashi, K. Shiokawa, and H. Kawano, The GEOTAIL Magnetic field experiment, J. Geomag. Geoelectr., 46, 7-21, 1994.

Krimigis, S. M. and E. T. Sarris, Energetic particle bursts in the earth's magnetotail, in Dynamics of the Magnetosphere, edited by S.-I. Akasofu, pp. 599-630, D. Reidel, Hingam, Mass., 1979.

Pavlos, G. P., E. T. Sarris, and N. Paschalidis, The growth rate and location of the acceleration of energetic particles inside the plasma sheet, Planet. Space Sci., 37, 503-516, 1989.

Richardson, I. G., C. J. Owen, J. A. Slavin, and T. T. von Rosenvinge, Energetic $(>0.2 \mathrm{MeV})$ electron bursts observed by ISEE 3 in the deep $\left(<240 \mathrm{R}_{\mathrm{E}}\right)$ geomagnetic tail, J. Geophys. Res., 98, 13,441-13,451, 1993.

Sarafopoulos, D. V. and E. T. Sarris, Transient field-aligned electric fields inside the plasma sheet inferred from measurements of energetic particles, Planet. Space Sci., 35, 991-995, 1987.

Sarafopoulos, D. V. and E. T. Sarris, Inverse velocity dispersion of energetic particle bursts inside the plasma sheet, Planet. Space Sci., 36, 1181-1199, 1988.

Sarris, E. T. and W. I. Axford, Energetic protons near the plasma sheet boundary, Nature, 277, 460, 1979.

Sarris, E. T., S. M. Krimigis, and T. P. Armstrong, Observations of magnetospheric bursts of high-energy protons and electrons at $35 \mathrm{R}_{\mathrm{E}}$ with IMP-7, J. Geophys. Res., 81, 2341-2355, 1976.

Taktakishvili, A. L. and L. M. Zelenyi, Inductive acceleration of protons and electrons in planetary magnetotails, in Plasma Astrophysics, ESA SP-285, Vol. II, edited by T. D. Guyenne and J. J. Hunt, pp. 227-232, 1988.

Taktakishvili, A. L. and L. M. Zelenyi, Temporal dispersion structure of the proton bursts in the Earth's magnetotail, in Plasma Astrophysics, ESA SP-311, edited by T. D. Guyenne, pp. 51-56, 1990.

Williams, D. J., Energetic ion beams at the edge of the plasma sheet: ISEE-1 observations plus a simple explanatory model, $J$. Geophys. Res., 86, 5507-5518, 1981.

Williams, D. J., R. W. McEntire, C. Schlemm, II, A. T. Y. Lui, G. Gloeckler, S. P. Christon, and F. Gliem, Geotail energetic particles and ion composition instrument, J. Geomag. Geoelectr., 46, 39-57, 1994.

Zelenyi, L. M., J. G. Lominadze, and A. L. Taktakishvili, Generation of energetic proton and electron bursts in planetary magnetotails, J. Geophys. Res., 95, 3883-3891, 1990. 